\title{
Testicular Teratoma
}

National Cancer Institute

\section{Source}

National Cancer Institute. Testicular Teratoma. NCI Thesaurus. Code C3877.

A non-seminomatous germ cell tumor arising from the testis. It is characterized by the presence of various tissues which correspond to the different germinal layers

(endoderm, mesoderm, and ectoderm). Testicular teratomas in children follow a benign

clinical course whereas in postpubertal patients may metastasize to other anatomic sites. 